\title{
Interval training during concurrent training optimizes cardiorespiratory adaptations in women
}

\section{Exercício intervalado durante o treinamento concorrente otimiza as adaptações cardiorrespiratórias em mulheres}

\author{
Rodrigo Ferrari \\ (1) https://orcid. org/0000-0003-1043-821X \\ Cristine Lima Alberton? \\ (1) https://orcid. org/0000-0002-5258-9406 \\ Stephanie Santana Pinto? \\ (1) https://orcid. org/0000-0003-4555-2717 \\ Eduardo Lusa Cadore' \\ (1) https://orcid.org/0000-0003-4397-9485 \\ Ronei Silveira Pinto ${ }^{\top}$ \\ (D) https://orcid.org/0000-0002-5827-5723 \\ Luiz Fernando Martins Kruel' \\ (D) https://orcid.org/0000-0002-9828-3437
}

Abstract - This study compared the effects of using continuous and interval aerobic exercise during concurrent training on cardiorespiratory adaptations in women. Thirty-two participants were randomly assigned into one of the following groups: continuous running and resistance training $(\mathrm{C}-\mathrm{RUN}, \mathrm{n}=10)$, interval running and resistance training (I-RUN, $\mathrm{n}=11)$, or control group that performed resistance training only $(\mathrm{RT}, \mathrm{n}=11)$. Each group trained twice a week during 11 weeks. Oxygen uptake corresponding to the first ventilatory threshold $\left(\mathrm{VO}_{2 \mathrm{VT} 1}\right)$, second ventilatory threshold $\left(\mathrm{VO}_{2 \mathrm{VT} 2}\right)$ and maximal effort $\left(\mathrm{VO}_{2 \max }\right)$ was measured in a maximal incremental test performed before and after training. Significant increases in $\mathrm{VO}_{2 V T 1}, \mathrm{VO}_{2 V T 2}$ and $\mathrm{VO}_{2 \max }$ were observed in all training groups. $\mathrm{VO}_{2 \mathrm{VT} 2}$ and $\mathrm{VO}_{2 \max }$ presented time-group interactions, indicating that the magnitude of the increase in these variables was dependent on the training group $\left(\mathrm{VO}_{2 \mathrm{TT}}: \mathrm{C}-\mathrm{Run}=6.6 \%\right.$, I-Run $=15.7 \%, \mathrm{RT}=1.7 \% ; \mathrm{VO}_{2}: \mathrm{C}-\mathrm{Run}=$ $7.2 \%, \mathrm{I}-\mathrm{Run}=14.3 \%, \mathrm{RT}=2.7 \%)$. The effect size observed for post-training values comparing $\mathrm{C}$-RUN and RT groups was $d=0.566$ for $\mathrm{VO}_{2 \mathrm{~T}}$ and $d=0.442$ for $\mathrm{VO}_{2}$. On the other hand, values of $d=0.949$ for $\mathrm{VO}_{2 \mathrm{VT} 2}$ and $d=1.189$ for $\mathrm{VO}_{2 \max }$ were verified between I-RUN and RT groups. In conclusion, the use of continuous and interval aerobic exercise during concurrent training improved different cardiorespiratory parameters in women, but in a greater magnitude when interval aerobic exercise was performed simultaneously to resistance training.

Key words: Cardiology; Exercise; Physical fitness; Oxygen uptake; Running.

Resumo - O presente estudo comparou os efeitos do uso de exercício aeróbio contínuo e intervalado durante o treinamento concorrente nas adaptaçôes cardiorrespiratórias de mulheres. Trinta e duas participantes foram randomizadas em três grupos de treinamento: corrida continua + treinamento resistido $(C-R U N, n=10)$, corrida intervalada + treinamento resistido $(I-R U N, n=11)$, e apenas treinamento resistido $(R T, n=11)$. Cada grupo treinou 2 vezes por semana ao longo de 11 semanas. Foi avaliado o consumo de oxigênio correspondente ao primeiro $\left(V O_{2 V T 1}\right)$ e segundo $\left(V O_{2 V T 2}\right)$ limiar ventilatório, bem como o consumo máximo de oxigênio $\left(V O_{2 m a}\right)$ antes e depois dos treinamentos. Foram encontrados aumentos significativos no $\mathrm{VO}_{2 V T 1}, V_{2 V T 2}$ eVO $\mathrm{VO}_{2 \max }$ ao final do periodo de treinamento nos três grupos. Ainda, foi encontrada interação tempo grupo nas variáveis $V \mathrm{~V}_{2 V T 2}$ e $V \mathrm{O}_{2 \mathrm{~m}}$ , sugerindo que a magnitude de alteração foi dependente do tipo de treinamento realizado $\left(V O_{2 V T 2}\right.$ : $C-R u n=6,6 \%, I-$ Run $\left.=15,7 \%, R T=1,7 \% ; V O_{2 \max }: C-R u n=7,2 \%, I-R u n=14,3 \%, R T=2,7 \%\right)$. Da mesma forma, diferentes tamanhos de efeito foram observados ao final do treinamento, de acordo com a intervenção realizada $\left(C-R U N\right.$ versus $R T d=0,566$ para o $V O_{2 V T 2}$ ed $=0,442$ para o $V O_{2 \text { max }}$ $I-R U N$ versus $R T d=0,949$ para o $V_{2 V T 2}$ e d $=1,189$ para o $\left.V O_{2 \text { max }}\right)^{2}$. Embora o uso de exercício aeróbio contínuo e intervalado durante o treinamento concorrente tenha sido efetivo em promover adaptaçôes cardiorrespiratórias em mulheres, os ganhos obtidos foram maiores quando o exercício intervalado foi associado ao exercício resistido.

Palavras-chave: Aptidão física; Consumo de oxigênio; Corrida; Cardiologia; Exercício físico.
1 Universidade Federal Rio Grande do Sul. Porto Alegre, RS. Brazil

2 Universidade Federal de Pelotas. Pelotas, RS. Brazil

Received: May 13, 2020 Accepted: October 09, 2020

How to cite this article

Ferrari R, Alberton CL, Pinto SS, Cadore EL, Pinto RS, Kruel LFM. Interval training during concurrent training optimizes cardiorespiratory adaptations in women. Rev Bras Cineantropom Desempenho Hum 2021; 23:e73867. DOl: http://doi. org/10.1590/1980-0037.2021v23e73867

Copyright: This work is licensed under a Creative Commons Attribution 4.0 International License. 


\section{INTRODUCTION}

The performance of regular resistance training during endurance training routine improves long-distance running performance ${ }^{1,2}$. In this context, simultaneous performance of resistance and aerobic exercises (i.e., concurrent training) has become an effective strategy to improve endurance performance in physically active ${ }^{3}$ and trained participants ${ }^{4}$. However, the exact amount of aerobic exercise during concurrent training needed to achieve favorable cardiorespiratory adaptations is not clear, since these changes depend on training intensity and volume. Current guidelines suggest $150 \mathrm{~min}$ per week and three days per week of aerobic exercise to reach this goal ${ }^{5}$. However, concurrent training has shown positive results on maximal oxygen uptake $\left(\mathrm{VO}_{2 \max }\right)$ with lower volumes in different populations $s^{6,7}$ of trained and untrained participants ${ }^{3,8}$.

Regarding optimal intensity, although higher intensities resulted in higher $\mathrm{VO}_{2 \max }$ improvements compared to lower intensities in young adults ${ }^{9}$, low to moderate intensities (i.e., $45 \% \mathrm{VO}_{2 \max }$ ) seems to be enough to improve $\mathrm{VO}_{2 \max }$, suggesting that different aerobic exercise intensities are effective for improving $\mathrm{VO}_{2 \max }{ }^{10}$. Continuous aerobic exercise (CA) as well as high-intensity interval training (HIIT) composed of intermittent bursts of vigorous activity interspersed by periods of rest or low-intensity exercise ${ }^{11}$, have been performed in order to improve $\mathrm{VO}_{2 \max }{ }^{12-14}$. HIIT has become a popular form of exercise due to its potentially large effects on exercise capacity and short time requirement ${ }^{15}$. A systematic review and meta-analysis evaluated the effects of CA and HIIT on $\mathrm{VO}_{2 \max }$ and suggested that HIIT improves $\mathrm{VO}_{2 \max }$ to a greater extent than $\mathrm{CA}^{16}$. However, studies comparing HIIT with CA are limited ${ }^{17}$, especially when performed simultaneously with resistance training ${ }^{18}$. Moreover, previous studies comparing HIIT vs. CA focused on $\mathrm{VO}_{2 \max }$ improvements are available, but there is scarce information about submaximal outcomes such as ventilatory thresholds, which are markers of endurance performance. Since concurrent training is able to improve these submaximal outcomes more than endurance training alone ${ }^{4}$, it seems very relevant to investigate what type of combination (i.e., Resistance + HIIT vs. Resistance + CA) would induce maximal and submaximal endurance outcomes. In addition, it would be interesting to investigate this issue in women, since there is lack of data comparing the effects of CA and HIIT on this population and men and women could respond differently to training ${ }^{19}$.

Based on the scarcity of data evaluating the effects of different aerobic exercise protocols and intensities during concurrent training, the aim of the present study was to compare the effects of using continuous and interval aerobic exercise during concurrent training on cardiorespiratory adaptations in women. Our hypothesis was that concurrent training using HIIT would induce higher cardiorespiratory improvement when compared to concurrent training using CA. 


\section{METHODS}

\section{Participants}

Thirty-two active young women, most of them physical education students who practice recreational sports but not engaged in any structured training program in the last 3 months, volunteered to participate in this study. All participants were free from any musculoskeletal, bone and joint, or cardiac and pulmonary diseases. Participants were informed about the procedures and potential risks and provided their written informed consent. Moreover, all participants were advised to maintain their normal dietary intake and daily physical activities throughout the study. The study was approved by the local Research Ethics Committee (Protocol number: 15406) and is in accordance with the Declaration of Helsinki. Participants' baseline characteristics are shown in Table 1.

Table 1. Participants' baseline characteristics (means \pm SD)

\begin{tabular}{llll}
\hline & $(\mathrm{RT} n=12)$ & $(\mathrm{C}-\mathrm{RUN} \mathrm{n}=10)$ & $(\mathrm{I}-\mathrm{RUN} \mathrm{n}=11)$ \\
\hline Age (years) & $23.5 \pm 2.5$ & $22.3 \pm 2.1$ & $24.3 \pm 5.0$ \\
Body mass $(\mathrm{kg})$ & $59.2 \pm 8.3$ & $59.8 \pm 6.7$ & $59.0 \pm 5.9$ \\
Height $(\mathrm{cm})$ & $165.8 \pm 6.5$ & $162.2 \pm 4.5$ & $166.7 \pm 4.0$ \\
$\%$ Fat mass & $24.7 \pm 4.0$ & $24.1 \pm 4.0$ & $23.9 \pm 4.2$ \\
1RM Leg Press $(\mathrm{kg})$ & $89.8 \pm 16.8$ & $100.5 \pm 16.3$ & $104.2 \pm 19.6$ \\
1RM Bench Press $(\mathrm{kg})$ & $29.5 \pm 6.5$ & $30.2 \pm 5.3$ & $32.0 \pm 5.5$ \\
\hline
\end{tabular}

Note. $\mathrm{C}-\mathrm{RUN}=$ continuous running and resistance training; $\mathrm{I}-\mathrm{RUN}=$ interval running and resistance training; $\mathrm{RT}=$ resistance training only

\section{Experimental Design}

To compare cardiorespiratory adaptations of continuous and interval running exercise during concurrent training in women, participants were randomly assigned into one of the following groups: continuous running and resistance training (C-RUN, n=10), interval running and resistance training (I-RUN, $n=11$ ), or resistance training only (RT, $n=11$ ). Participants took part in a training program that lasted 11 weeks, and trained twice a week on Mondays and Thursdays or on Tuesdays and Fridays. Before and after training, participants performed a cardiorespiratory test in order to determine $\mathrm{VO}_{2 \max }$, first (VT1) and second (VT2) ventilatory thresholds as well as the velocity and heart hate corresponding to these intensities.

Maximal strength and body composition were evaluated in order to characterize the study population. Body mass and height were measured using analog scale and stadiometer, respectively (Asimed, Camarate, Portugal). Body composition was assessed using skinfold technique (LANGE, Cambridge, United Kingdom). A seven-site skinfold equation was used to estimate body density, and body fat was subsequently calculated using the Siri equation ${ }^{20}$. Maximal dynamic strength was evaluated through the one-repetition maximal test (1RM) of leg press and bench press. After performing specific movements with 1 set of 15 repetitions with light load, the maximal load of each participant was determined with no more than 
five attempts with 4-min recovery between attempts. A detailed description of $1 \mathrm{RM}$ procedures has been described elsewhere ${ }^{21}$.

\section{Cardiorespiratory test}

Participants performed incremental test on treadmill (INBRAMED; Porto Alegre, Brazil). The protocol consisted of initial velocity of $5 \mathrm{~km} . \mathrm{h}^{-1}$ with $1 \%$ inclination during $2 \mathrm{~min}$. Subsequently, the velocity was increased every $1 \mathrm{~min}$ by increments of $1 \mathrm{~km} \cdot \mathrm{h}^{-1}$, and the inclination was maintained until participants reached their maximal effort. All incremental tests were supervised by a physician. The assessment was considered valid when some of the following criteria were met at the end of the test ${ }^{22}$ : estimated maximal heart rate was reached (220-age); oxygen uptake plateau with increase in treadmill velocity; respiratory exchange ratio greater than 1.15; maximum respiratory rate of at least 35 breaths per minute.

To evaluate ventilatory data, mixing-box-type portable gas analyzer (VO2000, MEDGRAPHICS; Ann Arbor, USA) previously calibrated according to manufacturer's instructions was used. The sampling rate of collected values was 1 sample every 10s, and data were acquired using the Aerograph software. VT1 was determined through the ventilation curve and confirmed by ventilatory curve equivalent for $\mathrm{O}_{2}$. VT2 was determined using ventilation curve corresponding to the point of exponential increase in ventilation in relation to the load. In addition, to confirm data, VT2 was determined using the $\mathrm{CO}_{2}$ ventilatory equivalent ${ }^{23}$. Three experienced and independent blind physiologists detected the corresponding points through visual inspection according to previously described criteria. Heart rate was continuously measured using Polar monitor (model FS1, Shanghai, CHI). Environmental conditions (e.g., room temperature at $22-24{ }^{\circ} \mathrm{C}$ ) were kept constant during tests. The test-retest reliability coefficient (ICC) was 0.87 for $\mathrm{VO}_{2 \max }$.

\section{Exercise training programs}

All exercise training groups trained two times per week throughout 11 weeks. The same resistance-training program was performed for all groups. At the beginning of each session, participants performed specific muscle stretching and warm-up with 1 set of 25 repetitions with light load to upper and lower body. The resistance-training program included seven exercises (leg press, knee extension, leg curl, bench press, inverted fly, upright row and sit-ups) performed until fatigue (repetition maximum - RM). During the first two weeks, participants performed two sets of 15-18 RM, progressing to three sets of 12-15 RM (weeks 3-5), three sets of 10-12 RM (weeks 6-8), and finally three sets of 8-10 RM (weeks 9-11). RM tests were performed for all exercises at the beginning of each period (i.e., week 1, 3,6 and 9). The workload was adjusted when repetitions performed were either under or above established repetitions. The recovery time between sets was 120 seconds.

Concurrent training groups performed running and resistance exercises during the same day/session, starting with running and immediately 
followed by resistance exercises. The intra-session order based on previous study that found greater improvement in endurance performance and aerobic capacity when endurance training preceded resistance training was adopted ${ }^{3}$.

Aerobic sessions in both groups lasted the same time, beginning with $20 \mathrm{~min}$ in the first two weeks, progressing to 25 min (weeks 3-8), and finishing with $30 \mathrm{~min}$ (weeks 9-11). These groups were differentiated by the aerobic training intensity, in which C-RUN performed CA at HR equivalent to $95 \%$ of $\mathrm{VT}_{2}(\mathrm{i} 95 \% \mathrm{VT} 2$ ) and I-RUN performed HIIT consisting of one-minute bouts at velocity corresponding to $\mathrm{VO}_{2 \max }\left(\mathrm{iVO}_{2 \max }\right)$, with one minute of active recovery bouts at $50 \%$ of $\mathrm{iVO}_{2 \max }$. All training sessions were carefully supervised by at least 2 experienced personal trainers.

\section{Statistical Analyses}

Descriptive statistics was used to analyze collected data presented as mean \pm SD. Shapiro-Wilk and Levene tests were used to verify normal data distribution and homogeneity. Repeated measures ANOVA with group factor was used to analyze data. When applicable, Bonferroni's post hoc tests were used to find significant differences. The effect size was determined by partial $\eta^{2}$ for each main effect and interaction, as well as by Cohen's $d$ for the comparison of post-training values. Alpha of $\mathrm{p}<0.05$ was adopted. SPSS software version 20.0 was used in analyses.

\section{RESULTS}

All participants complied with at least $90 \%$ of training with no difference among groups in the number of sessions performed. In addition, no significant differences among groups at baseline characteristics were found $(\mathrm{p}>0.05)$.

Main effect of training was observed for $\mathrm{VO}_{2 \mathrm{VT} 1}(\mathrm{p}<0.001$, partial $\left.\eta^{2}=0.402\right), \mathrm{VO}_{2 \mathrm{VT} 2}\left(\mathrm{p}=0.004\right.$, partial $\left.\eta^{2}=0.263\right)$ and $\mathrm{VO}_{2 \max }(\mathrm{p}=0.001$, partial $\eta^{2}=0.348$ ). The main effect of group was not significant for any variable $(p>0.05)$. However, the time-group interaction resulted in trend for $\mathrm{VO}_{2 \mathrm{VT} 2}\left(\mathrm{p}=0.093\right.$, partial $\left.\eta^{2}=0.162\right)$ and $\mathrm{VO}_{2 \max }\left(\mathrm{p}=0.085\right.$; partial $\eta^{2}$ $=0.167)$, indicating that the magnitude of the increase in these variables was dependent on the training group $\left(\mathrm{VO}_{2 \mathrm{VT} 2}: \mathrm{C}-\mathrm{Run}=6.6 \%\right.$, I-Run $=$ $\left.15.7 \%, \mathrm{RT}=1.7 \% ; \mathrm{VO}_{2 \max }: \mathrm{C}-\mathrm{Run}=7.2 \%, \mathrm{I}-\mathrm{Run}=14.3 \%, \mathrm{RT}=2.7 \%\right)$, which results are shown in Figure 1.

In addition, the effect size observed for post training values in the comparison between C-RUN and RT groups was $d=0.566$ for $\mathrm{VO}_{2 \mathrm{VT} 2}$, considered as a moderate effect, and $d=0.442$ for $\mathrm{VO}_{2 \max }$, considered as a small effect. On the other hand, values of $d=0.949$ for $\mathrm{VO}_{2 \mathrm{VT} 2}$ and $d$ $=1.189$ for $\mathrm{VO}_{2 \max }$ were verified between I-RUN and RT groups, both considered as a large effect. 

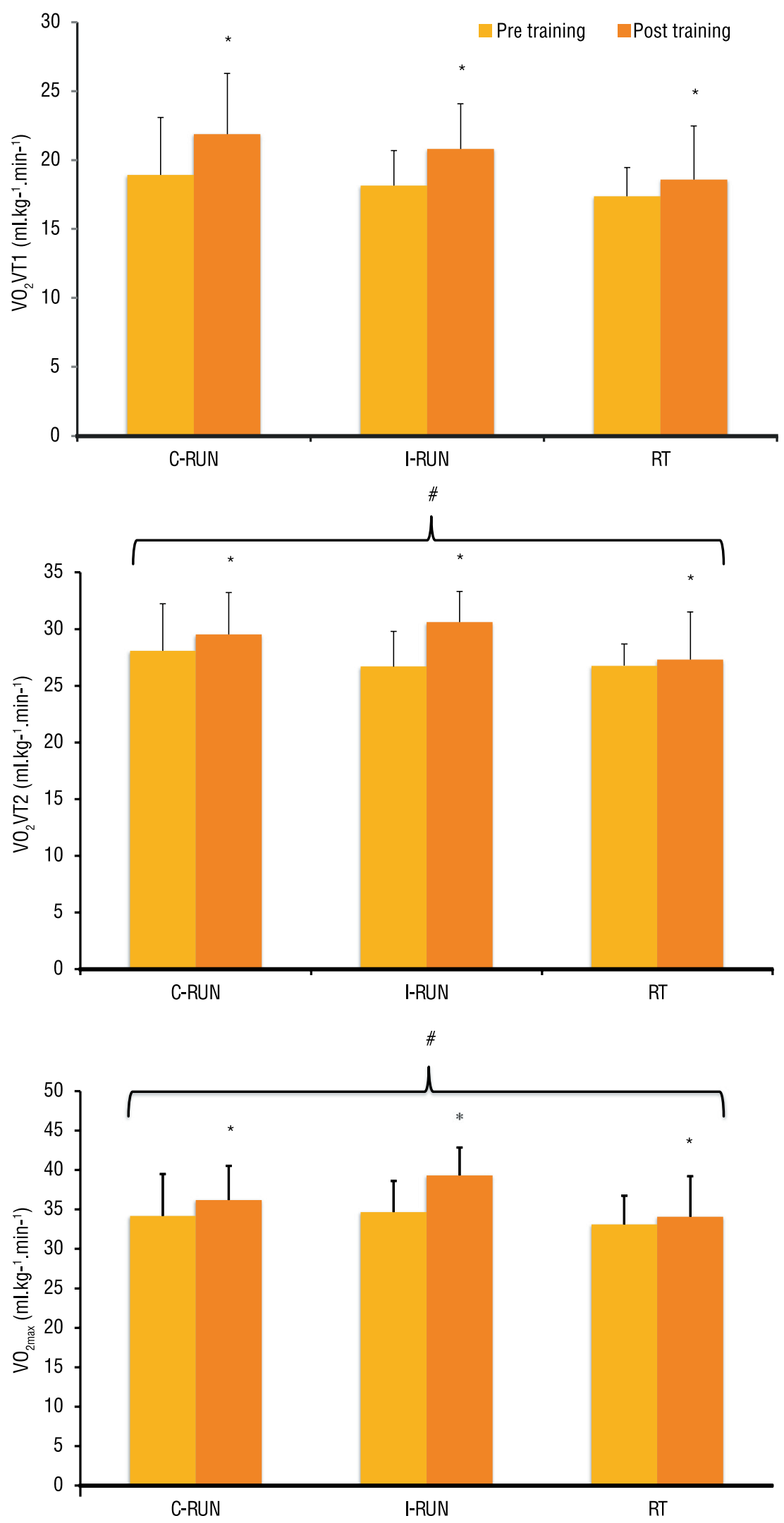

Figure 1. Oxygen uptakes corresponding to the first ventilatory threshold (VO,VT1), second ventilatory threshold $\left(\mathrm{VO}_{2} \mathrm{VT2}\right)$ and maximal oxygen uptake $\left(\mathrm{VO}_{2 \max }\right)$ before and after training for continuous running and resistance training (C-RUN), interval running and resistance training (I-RUN), or resistance training only (RT) groups. * Different from corresponding pre training value $(p<0.05)$; \# Trend toward significant time vs. group interaction for $\mathrm{VO}_{2} \mathrm{VT2}(\mathrm{P}=0.093)$ and $\mathrm{VO}_{2 \max }(\mathrm{P}=0.085)$ 


\section{DISCUSSION}

The primary finding of the present study was the positive effects of concurrent training on different cardiorespiratory parameters, especially when HIIT was performed simultaneously to resistance training. Such results are in agreement to our hypothesis, highlighting the efficiency of HIIT to promote $\mathrm{VO}_{2 \max }$ and $\mathrm{VO}_{2 \mathrm{VT} 2}$ improvements during concurrent exercise. In a recent study, $\mathrm{VO}_{2 \max }$ improvement after 8 weeks of HIIT was similar to conventional CA in young adults ${ }^{17}$. It should be highlighted that one of the HIIT protocols in the abovementioned study was time efficient (i.e., $4 \mathrm{~min}$ ), while the other one was time-matched to CA (i.e., $20 \mathrm{~min}$ ), all of them performing aerobic exercise alone. In the present study, in which HIIT and CA were performed simultaneously to resistance exercise using a timematched protocol, results revealed larger effects on $\mathrm{VO}_{2 \max }$ from HIIT when compared to conventional CA (C-RUN 7\% and I-RUN 14\%). It should be considered that exercising at high relative intensity seems to induce larger beneficial adaptation in the cardiovascular system ${ }^{9,24}$ as well as the optimal training intensity that elicits aerobic capacity is highly dependent on the initial training status of participants ${ }^{25}$. In this context, the different results between the abovementioned study ${ }^{17}$ and the present study could be associated to the previous status of participants (sedentary vs. physically active), and that training at or near $\mathrm{iVO}_{2 \max }$ may be more effective to enhance $\mathrm{VO}_{2 \max }$ in physically active women. The importance of designing effective exercise programs and HIIT may be particularly critical for maximizing the aerobic capacity improvement. Moreover, when using different aerobic (running vs cycling) or resistance exercises targeting strength, hypertrophic or power, training adaptations may be quite different ${ }^{26}$, and these protocol differences could partially explain these discrepancies.

Minimum of three sessions per week has been suggested to increase cardiorespiratory fitness after aerobic training ${ }^{5}$. The present study demonstrated positive results on $\mathrm{VO}_{2 \mathrm{VT} 1}, \mathrm{VO}_{2 \mathrm{VT} 2}$ and $\mathrm{VO}_{2 \max }$ performing only two sessions per week of 20-30 min of aerobic exercise in women. Our results corroborate those of Chtara et al. ${ }^{3}$, who demonstrated that two sessions per week of concurrent training (HIIT + resistance exercise) increase $\mathrm{VO}_{2 \max }$ in physically active men. A unique characteristic of the present study was the comparison of different aerobic training intensities during concurrent training. Both I-RUN and C-RUN improved $\mathrm{VO}_{2 \max }$ and $\mathrm{VO}_{2 \mathrm{VT} 2}$ values at magnitudes comparable to those observed in other studies ${ }^{1,3}$. In addition, the use of HIIT during concurrent training confirms additional benefits of this training when compared to CA. Although there was an increase in the RT group, as expected, this improvement was smaller than that observed in C-RUN and I-RUN groups. Indeed, some studies have shown that resistance training can provide slight improvement in maximum aerobic capacity in individuals not previously trained in aerobic exercise ${ }^{27}$. This increased performance has been attributed in part to improved movement economy, since the increase in strength results in greater recruitment of type I fibers 
for the same submaximal load, better muscular coordination and therefore better mechanical efficiency, which reduces $\mathrm{VO}_{2}$ for the same submaximal intensity and may have some influence on maximal aerobic capacity ${ }^{28}$.

It could be observed that both concurrent training programs were effective to improve the cardiorespiratory capacity in young women (C-RUN $\approx 7 \%$, and I-RUN $\approx 14 \%$ ). Such gains were also found in studies that evaluated the effects of resistance training programs as an additive to an aerobic running program in young individuals ${ }^{1}$. After a 10 -week training period performed three times per week, increases in $\mathrm{VO}_{2 \max }$ values of $\approx 13 \%$ and $\approx 7 \%$ for the concurrent training and aerobic training groups, respectively, were found in young women ${ }^{1}$. It is important to highlight that in the present study, participants performed only two aerobic exercise sessions of 20-30 min per week, whereas in the abovementioned study ${ }^{1}$, they performed three sessions of 60 min per week, demonstrating the efficiency of lower frequency concurrent training performed in the present study in this population.

Significant improvements were also found for $\mathrm{VO}_{2 \mathrm{VT} 1}$ and $\mathrm{VO}_{2 \mathrm{VT} 2}$ after 11 weeks of training, and the use of the HIIT protocol demonstrated additional improvements in $\mathrm{VO}_{2 \mathrm{VT} 2}$. These results are in agreement with literature regarding the use of $\mathrm{HIIT}^{29,30}$ and may be related to the use of intensities close to $\mathrm{VO}_{2 \max }$ to promote optimal benefits in $\mathrm{VO}_{2 \mathrm{VT} 2}$, which is an important parameter related to running performance ${ }^{13}$. The mechanisms believed responsible for the performance improvement associated with training at $\mathrm{iVO}_{2 \max }$ are an improvement in critical power improving cardiac function $^{24}$, a reduction in oxygen deficit with less anaerobic contribution at the onset of exercise and increases in ventilatory and lactate thresholds ${ }^{13,29}$.

The present study has strengths and limitations. One could suggest that resistance training performed by I-RUN and C-RUN could have some influence in endurance training adaptations, since participants performed concurrent training. However, all training groups performed the same resistance-training program, and a possible influence would be the same among groups. In addition, the prescription of resistance training avoided the possibility that participants could engage themselves in some resistance-training program outside our intervention, which could have influenced results. Another possible limitation of the present study was the assessment of participants after only 11 weeks of training, and these results cannot be extrapolated to longer training interventions. On the other hand, this is the first study to compare the effects of concurrent training composed of CA and HIIT on the cardiorespiratory adaptations in young women, which is a strenght and results bring new information regarding concurrent training prescription in this population.

\section{CONCLUSION}

The use of continuous and interval aerobic exercise during concurrent training improved different cardiorespiratory parameters in women, but in a greater magnitude when interval aerobic exercise was performed simulta- 
neously to resistance training. Based on the above, our results suggest that concurrent training performed twice a week using continuous or interval running promotes improvements in $\mathrm{VO}_{2 \mathrm{VT} 1}, \mathrm{VO}_{2 \mathrm{VT} 2}$ and $\mathrm{VO}_{2 \max }$, especially when HIIT is performed during concurrent training.

It should also be pointed out that, even in untrained women, HIIT intervention performed in the present study was feasible and quite effective to optimize cardiorespiratory gains in these individuals, even using volumes increasing from 20 to 30 minutes per session. These improvements in different cardiorespiratory parameters highlight the efficiency of low frequency concurrent exercise interventions, with lower time expenditure, which may facilitate training adherence.

\section{Acknowledgements}

We gratefully acknowledge to all the subjects who participated in this research and made this project possible.

\section{COMPLIANCE WITH ETHICAL STANDARDS}

\section{Funding}

The study was partially supported by CAPES and CNPq, Brazilian Government Associations.

\section{Ethical approval}

Ethical approval was obtained from the local Human Research Ethics Committee (UFRGS) and protocol (No. 15406) was written in accordance with standards set by the Declaration of Helsinki.

\section{Conflict of interest statement}

The authors have no conflict of interests to declare.

\section{Author Contributions}

Conceived and designed experiments: RF and LF. Performed experiments: RF, CL. Analyzed data: CL and SS. Contributed with reagents/materials/ analysis tools: RS, EL. Wrote the paper: RF, CL, SS.

\section{REFERENCES}

1. Kelly CM, Burnett AF, Newton MJ. The effect of strength training on threekilometer performance in recreational women endurance runners. J Strength Cond Res 2008; 22: 396-403.

2. Berryman N, Mujika I, Arvisais D, Roubeix M, Binet C, Bosquet L. Strength Training for Middle- and Long-Distance Performance: A Meta-Analysis. Int J Sports Physiol Perform 2018; 13: 57-63.

3. Chtara M, Chamari K, Chaouachi M, Koubaa D, Feki Y, Millet GP, Amri M. Effects of intra-session concurrent endurance and strength training sequence on aerobic performance and capacity. Br J Sports Med 2005;39(8):555-60.

4. Paavolainen L, Hakkinen K, Hamalainen I, Nummela A, Rusko H. Explosivestrength training improves $5-\mathrm{km}$ running time by improving running economy and muscle power. J Appl Physiol 1999; 86(5): 1527-33. 
5. Garber CE, Blissmer B, Deschenes MR, Franklin BA, Lamonte MJ, Lee I, et al. American College of Sports Medicine position stand. Quantity and quality of exercise for developing and maintaining cardiorespiratory, musculoskeletal, and neuromotor fitness in apparently healthy adults: guidance for prescribing exercise. Med Sci Sports Exerc 2011; 43(7): 1334-59.

6. Häkkinen K, Alen M, Kraemer WJ, Gorostiaga E, Izquierdo M, Rusko H, et al. Neuromuscular adaptations during concurrent strength and endurance training versus strength training. Eur J Appl Physiol 2003;89(1):42-52.

7. Karavirta L, Tulppo MP, Laaksonen DE, Nyman K, Laukkanen RT, Kinnunen $\mathrm{H}$. Heart rate dynamics after combined endurance and strength training in older men. Med Sci Sports Exerc 2009; 41(7): 1436-43.

8. Ferrari R, Kruel LF, Cadore EL, Alberton CL, Izquierdo M, Conceição M, et al. Efficiency of twice weekly concurrent training in trained elderly men. Exp Geront 2013; 48(11): 1236-42.

9. Gormley SE, Swain DP, High R, Dowling EA, Kotipalli US, Gandrakota R. Effect of intensity of aerobic training on VO2max. Med Sci Sports Exerc 2008; 40(7): 1336-43.

10. Swain DP, Franklin BA. $\mathrm{VO}(2)$ reserve and the minimal intensity for improving cardiorespiratory fitness. Med Sci Sports Exerc 2002; 34(1): 152-7.

11. Gibala MJ, Little JP, Macdonald MJ, Hawley JA. Physiological adaptations to low-volume, high-intensity interval training in health and disease. J Physiol 2012; 590(5): 1077-84.

12. Holloszy JO, Coyle EF. Adaptations of skeletal muscle to endurance exercise and their metabolic consequences. J Appl Physiol 1984; 56(4): 831-8.

13. Billat LV. Interval training for performance: a scientific and empirical practice. Special recommendations for middle- and long-distance running. Part I: aerobic interval training. Sports Med 2001; 31(1): 13-31.

14. Midgley AW, McNaughton LR, Wilkinson M. Is there an optimal training intensity for enhancing the maximal oxygen uptake of distance runners?: empirical research findings, current opinions, physiological rationale and practical recommendations. Sports Med 2006; 36(2): 117-32.

15. MacInnis MJ, Gibala MJ. Physiological adaptations to interval training and the role of exercise intensity. J Physiol 2017;595(9):2915-2930.

16. Milanovic Z, Sporis G, Weston M. Effectiveness of High-Intensity Interval Training (HIT) and Continuous Endurance Training for VO2max Improvements: A Systematic Review and Meta-Analysis of Controlled Trials. Sports Med 2015; 45(10): 1469-81.

17. Foster C, Farland CV, Guidotti F, Harbin M, Roberts B, Schuette J, et al. The Effects of High Intensity Interval Training vs Steady State Training on Aerobic and Anaerobic Capacity. J Sports Sci Med 2015; 14(4): 747-55.

18. Fyfe JJ, Bartlett JD, Hanson ED, Stepto NK, Bishop DJ. Endurance Training Intensity Does Not Mediate Interference to Maximal Lower-Body Strength Gain during Short-Term Concurrent Training. Front Physiol 2016; 7: 487.

19. Bagley L, Slevin M, Bradburn S, Liu D, Murgatroyd C, Morrissey G, et al. Sex differences in the effects of 12 weeks sprint interval training on body fat mass and the rates of fatty acid oxidation and VO2max during exercise. BMJ Open Sport Exerc Med 2016; 2(1):e000056.

20. Jackson AS, Pollock ML, Ward A. Generalized equations for predicting body density of women. Med Sci Sports Exerc 1980; 12(3): 175-81.

21. Silva RF, Cadore EL, Kothe G, Guedes M, Alberton CL, Pinto SS, et al. Concurrent training with different aerobic exercises. Int J Sports Med 2012; 33(8): 627-34.

22. Bassett DR Jr., Howley ET. Limiting factors for maximum oxygen uptake and determinants of endurance performance. Med Sci Sports Exerc 2000; 32(1): 70-84.

23. Wasserman K, Whipp BJ, Koyl SN, Beaver WL. Anaerobic threshold and respiratory gas exchange during exercise. J Appl Physiol 1973; 35(2): 236-43. 
24. Wisloff U, Ellingsen O, Kemi OJ. High-intensity interval training to maximize cardiac benefits of exercise training? Exerc Sport Sci Rev 2009; 37(3): 139-46.

25. Gibala MJ, Gillen JB, Percival ME. Physiological and health-related adaptations to low-volume interval training: influences of nutrition and sex. Sports Med 2014; 44 (Suppl 2): S127-37.

26. Taipale RS, Mikkola J, Nummela AT, Sorvisto J, Nyman K, Kyröläinen H, et al. Combined strength and endurance session order: differences in force production and oxygen uptake. Int J Sports Physiol Perform 2015; 10(4): 418-25.

27. Gettman LR, Culter LA, Strathman TA. Physiologic changes after 20 weeks of isotonic vs isokinetic circuit training. J Sports Med Phys Fitness 1980; 20(3): 265-74.

28. Tanaka H, Swensen T. Impact of resistance training on endurance performance. A new form of cross-training? Sports Med 1998; 25(3): 191-200.

29. Burke J, Thayer R, Belcamino M. Comparison of effects of two interval-training programmes on lactate and ventilatory thresholds. Br J Sports Med 1994; 28(1): 18-21.

30. Laursen PB, Shing CM, Peake JM, Coombes JS, Jenkins DG. Influence of highintensity interval training on adaptations in well-trained cyclists. J Strength Cond Res 2005; 19(3): 527-33.

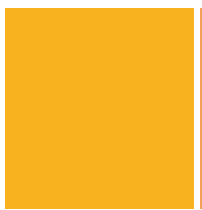

Corresponding author

Rodrigo Ferrari, PhD

Hospital de Clínicas de Porto Alegre:

Rua Ramiro Barcelos 2350, 90035-903

Porto Alegre, RS, Brazil.

Email: rod.ferrari84@gmail.com 